\title{
Inelastic alpha scattering studies of the low-energy octupole resonance*
}

\author{
J. M. Moss, D. R. Brown, D. H. Youngblood, C. M. Rozsa, and J. D. Bronson \\ Cyclotron Institute and Physics Department, Texas A\&M University, College Station, Texas 77843
}

(Received 21 February 1978)

\begin{abstract}
Beams of 96 and $115 \mathrm{MeV}$ a particles have been used to study the distribution of isoscalar octupole strength in 18 nuclei from ${ }^{40} \mathrm{Ca}$ to ${ }^{208} \mathrm{~Pb}$. A prominent broad peak $(\Gamma \sim 2.5 \mathrm{MeV})$ is observed at $E_{x} \sim 30 / A^{1 / 3}$ $\mathrm{MeV}$ in nuclei from ${ }^{66} \mathrm{Zn}$ to ${ }^{197} \mathrm{Au}$. No broad peak is observed in this excitation energy range in ${ }^{208} \mathrm{~Pb}$ or ${ }^{40} \mathrm{Ca}$ and lighter nuclei. The oscillatory angular distributions of the $\left(\alpha, \alpha^{\prime}\right)$ reaction exciting this peak are in excellent agreement with $l=3$ distorted-wave Born approximation calculations. Studies of the angular range from $\sigma_{\mathrm{lab}}=3.5^{\circ}$ to $6^{\circ}$ in ${ }^{116} \mathrm{Sn}$ indicate very little contribution from $l=1$ strength. Energy- weighted sum rule fractions for this low-energy octupole resonance are generally in the range from $15 \%$ to $20 \%$; this corresponds to $1 / 2$ to $2 / 3$ of the expected $1 \hbar \omega$ octupole strength. The overall distribution of octupole strength in spherical nuclei, including the absence of the low-energy octupole resonance in ${ }^{40} \mathrm{Ca}$ and ${ }^{208} \mathrm{~Pb}$, is in very good agreement with random-phase approximation calculations. The low-energy octupole resonance undergoes a pronounced change in structure in soft-vibrational and deformed nuclei. Theoretical calculations for the low-energy octupole resonance in ${ }^{154} \mathrm{Sm}$ account qualitatively for the data.
\end{abstract}

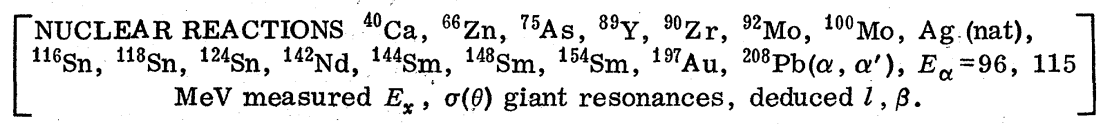

\section{INTRODUCTION}

In recent years considerable progress has been made in understanding giant multipole resonances in nuclei. ${ }^{1-5}$. It is now well established that the isovector giant dipole (GDR) and isoscalar giant quadrupole resonances (GQR) are consistent features of all but the very lightest nuclei. The knowledge of the response functions of other multipoles is not as complete (we confine the discussion to giant electric resonances). Evidence from $(p, \gamma)$ reactions suggests an isovector GQR in the $\mathrm{Pb}$ region, ${ }^{5}$ while recent $\left(\alpha, \alpha^{\prime}\right)$ work has identified the long sought after isoscalar giant monopole resonance in ${ }^{208} \mathrm{~Pb}$ and ${ }^{144} \mathrm{Sm}$. ${ }^{4}$ The present work deals with a giant resonance-like structure in the isoscalar $E 3$ response function at an excitation energy of $30 / A^{1 / 3} \mathrm{MeV}$. This low-energy octupole resonance (LEOR), the subject of an earlier publication, ${ }^{3}$ was found to exhaust $20-30 \%$ of the isoscalar E3 strength allowed by the energyweighted sum rule (EWSR) in medium mass nuclei. It was shown that the LEOR is in excellent accord with the properties of the lower of the two isoscalar giant octupole resonances expected from the schematic model and that essentially all of the $1 \hbar \omega$ octupole strength is accounted for in the mass region $A=90$ to $A=148$. This work is an extension of that reported in Ref. 3 to a wider range of target nuclei. With the aid of a larger body of experimental data we have attempted to understand the gross features of the LEOR in terms of random phase approximation (RPA) calculations of the structure of this state.
The $\left(\alpha, \alpha^{\prime}\right)$ reaction, employed in the present study of the LEOR, has several advantages in giant resonance investigations. Among them are the following: (1) Only isoscalar $(\Delta T=0)$ excitations are strongly excited; (2) diffraction-like angular distributions are characteristic of the angular momentum transfer thereby allowing $J^{\pi}$ to be assigned to the states excited; (3) considerable phenomenology exists on the extraction of isoscalar transition strengths, particularly with regard to their sensitivity to the optical model and their relationship to electromagnetic transition strengths. ${ }^{6}$ The principle disadvantages of hadronic inelastic scattering, including the $\left(\alpha, \alpha^{\prime}\right)$ reaction, are the large and poorly understood "background" which arises from real reaction processes, and the fact that many different multipolarities can be excited in the reaction.

\section{EXPERIMENTAL PROCEDURE AND DATA ANALYSIS}

Beams of 96 and $115 \mathrm{MeV} \alpha$ particles provided by the Texas A\&M cyclotron were used to bombard self-supporting foils from 0.5 to $10 \mathrm{mg} / \mathrm{cm}^{2}$ thickness. Two $\Delta E-E$ detector telescopes were employed to detect the scattered $\alpha$ particles except for the extreme forward angle data of ${ }^{116} \mathrm{Sn}$ where an Enge split-pole spectrograph was employed. The details of the data taking systems including procedures for energy calibration and elimination of extraneous experimental backgrounds are discussed thoroughly in Refs. 2 and 4. Table I lists the beam energy and number of angles for each of 
TABLE I. Targets studied.

\begin{tabular}{ccc}
\hline \hline Target & $E_{\alpha}(\mathrm{MeV})$ & No. of Angles \\
\hline${ }^{40} \mathrm{Ca}$ & 96,115 & 18,8 \\
${ }^{66} \mathrm{Zn}$ & 115 & 4 \\
${ }^{75} \mathrm{As}$ & 115 & 10 \\
${ }^{89} \mathrm{Y}$ & 115 & 2 \\
${ }^{90} \mathrm{Zr}$ & 96 & 15 \\
${ }^{92} \mathrm{Mo}$ & 115 & 2 \\
${ }^{96} \mathrm{Mo}$ & 115 & 2 \\
${ }^{100} \mathrm{Mo}$ & 115 & 2 \\
${ }^{N a t} \mathrm{Ag}$ & 115 & 4 \\
${ }^{116} \mathrm{Sn}$ & 115 & 22 \\
${ }^{118} \mathrm{Sn}$ & 115 & 2 \\
${ }^{12} \mathrm{Sn}$ & 115 & 15 \\
${ }^{142} \mathrm{Nd}$ & 115 & 10 \\
${ }^{144} \mathrm{Sm}$ & 115 & 10 \\
${ }^{148} \mathrm{Sm}$ & 115 & 13 \\
${ }^{154} \mathrm{Sm}$ & 115 & 14 \\
${ }^{197} \mathrm{Au}$ & 115 & 8 \\
${ }^{208} \mathrm{~Pb}$ & 96,115 & 17,18 \\
\hline \hline
\end{tabular}

the targets studied. The overall energy resolution was 200-150 MeV.

As in studies of other giant resonances with hadronic probes, care must be taken in the subtrac-

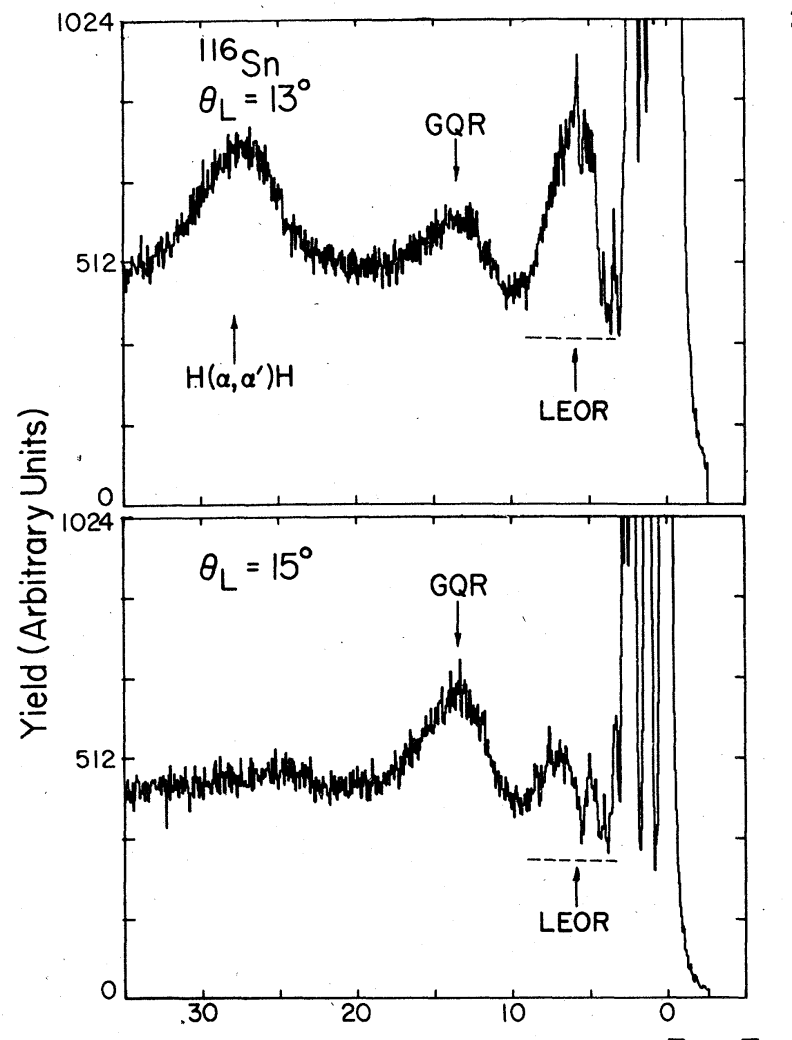

tion of the real continuum underlying the resonance in question. Since the origin of the continuum is not well understood, empirical procedures must be applied in order to subtract it. In the present work we utilize the fact that the continuum and resonance have very different angular dependences in order to separate them. ${ }^{2,3}$ This procedure obviously works best when the resonance is a relatively narrow structure with a simple shape. Figure 1 shows the two extremes encountered in the present work. In ${ }^{116} \mathrm{Sn}$ a large symmetric LEOR can be observed at several maxima and minima; the spectra at the minima allow one to obtain the background with little interference from the resonance itself. The case of ${ }^{75} \mathrm{As}$ is considerably less clear. Although the observation of a structure having maxima and minima within $0.5^{\circ}$ of those expected for $l=3$ is a clear indication of octupole strength, the uncertainty of exactly where, how much, and in what shape, is very large. This case is close to the limit of sensitivity of the $\left(\alpha, \alpha^{\prime}\right)$ reaction to separate multipole strength from the continuum.

In most of the nuclei studied the peak shape of the resonance remaining after continuum subtrac-

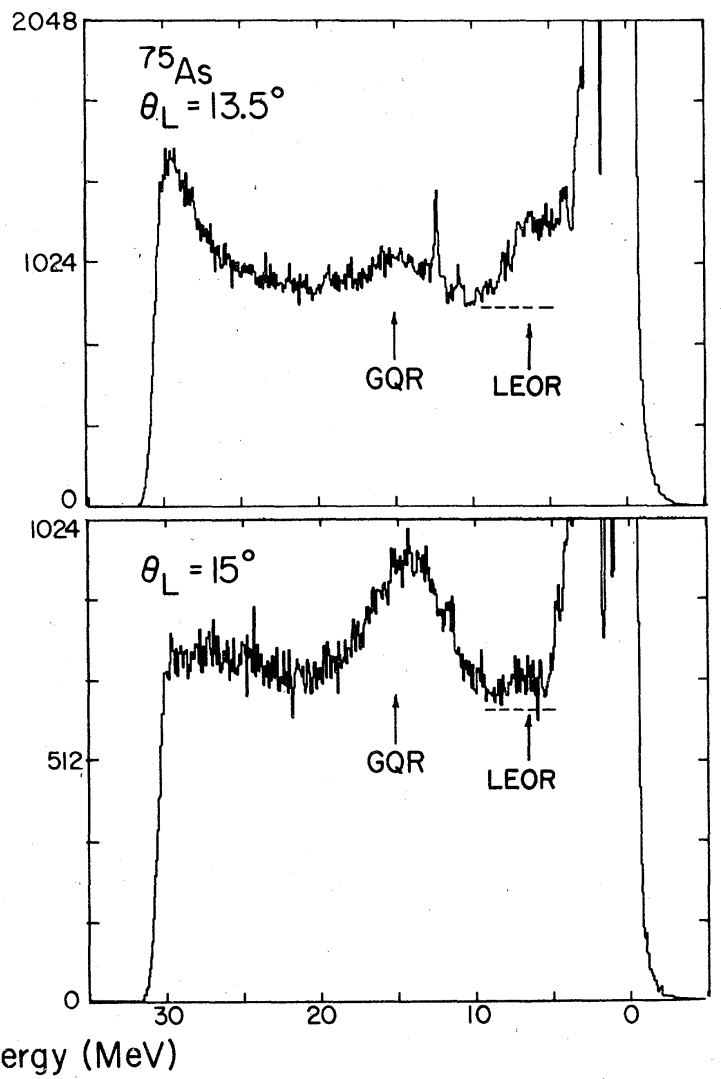

FIG. 1. Spectra of $\left(\alpha, \alpha^{\prime}\right)$ reaction on ${ }^{116} \mathrm{Sn}$ and ${ }^{75} \mathrm{As}$ at angles corresponding to maxima and minima in the cross section for $l=3$ transfer. 
tion had the form of a broad ( 2 to $3 \mathrm{MeV}$ ) Gaussian of Lorentzian distribution superimposed with narrow states whose angular distributions were most frequently not the same as that of the broad peak. In the deformed nucleus ${ }^{15} \mathrm{Sm}$ two broad peaks were recognizable at most angles. The data reduction consisted of fitting the continuum-subtracted spectrum with a series of broad and narrow Gaussian lines. The angular distributions of all components were then examined. Alternately, the narrow lines were subtracted from the spectrum and a simple summation was performed to get the strength of the broad peak. Similar results were obtained in cases where both methods were used. In general the LEOR is above the $p$ and $\alpha$ separation energies and roughly at the same position as the neutron separation evergy. It is likely in some cases that a substantial increase in energy would resolve the LEOR into numerous discrete states.

Figure 2 shows a spectrum near the expected
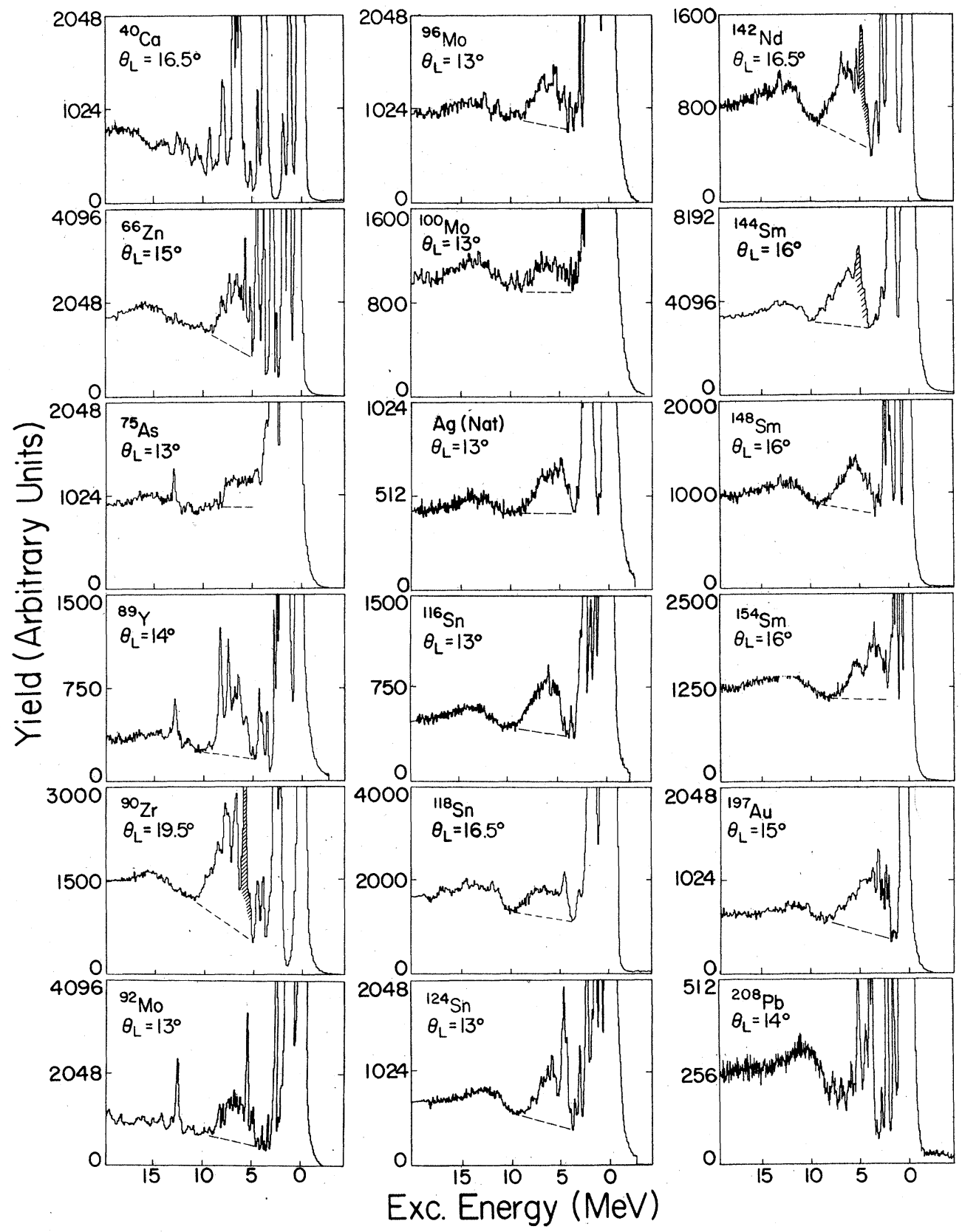

FIG. 2. Spectra of the $\left(\alpha, \alpha^{\prime}\right)$ reaction at a maximum for $l=3$. The LEOR, when present, is above the dashed background line. The shaded areas are contributions from states with $J^{\pi} \neq 3^{-}$. 
TABLE II. Optical potentials for the $\left(\alpha, \alpha^{\prime}\right)$ reactions.

\begin{tabular}{rccccc}
\hline $\begin{array}{c}E_{\alpha} \\
(\mathrm{MeV})\end{array}$ & Nucleus & $V(\mathrm{MeV})$ & $W(\mathrm{MeV})$ & $\boldsymbol{r}_{o}(\mathrm{fm})$ & $a(\mathrm{fm})$ \\
\hline 96 & ${ }^{90} \mathrm{Zr}$ & 27.7 & 1.50 & 0.7 \\
115 & ${ }^{116} \mathrm{Sn},{ }^{124} \mathrm{Sn}$ & 65.7 & 40.9 & 1.38 & 0.74 \\
115 & ${ }^{142} \mathrm{Nd},{ }^{148} \mathrm{Sm},{ }^{154} \mathrm{Sm}$ & 59.3 & 40.5 & 1.39 & 0.79 \\
115 & ${ }^{197} \mathrm{Au}$ & 89.3 & 52.7 & 1.35 & 0.71 \\
\hline \hline
\end{tabular}

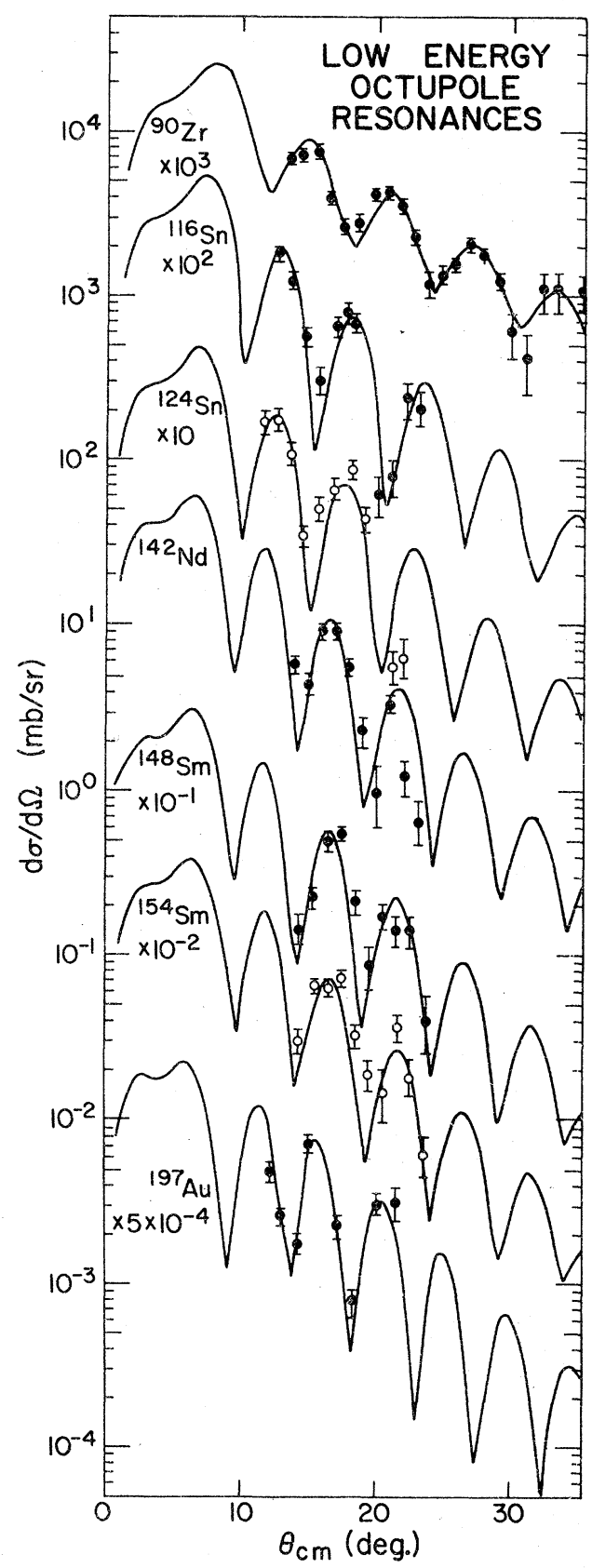

FIG. 3. Angular distributions for the LEOR in various nuclei. The $l=3$ DWBA fits are normalized to experiment. maximum for $l=3$ for all the nuclei studied. The dotted lines indicate the level of continuum subtracted from each spectrum and the approximate limit of the peak integration. Among the notable features of Fig. 2 is the absence of a welldefined broad peak in ${ }^{40} \mathrm{Ca}$ and ${ }^{208} \mathrm{~Pb}$. This phenomenon is discussed in Sec. V B.

\section{DISTORTED-WAVE BORN APPROXIMATION SUM-RULE ANALYSIS}

The application of the collective model-DWBA (distorted-wave Born approximation) analysis to giant resonances has been described in detail by Satchler. ${ }^{7}$ An isoscalar excitation of multipolarity $l(l \geqslant 2)$ located at an excitation energy $E$, which completely exhausts the EWSR, has a deformation length

$$
(\beta R)^{2}=l(2 l+1)\left(\hbar^{2} / 2 m E\right)(4 \pi / 3 A) .
$$

DWBA calculations using optical-model parameters (Table II) derived from elastic scattering data were performed using the code DWUCK. ${ }^{8}$ As has been noted previously many times ${ }^{2,4,6}$ the DWBA predictions are not sensitive to the opticalpotential set used so long as it reproduces the elastic scattering data. The normalized fits to selected LEOR's are shown in Fig. 3. From the DWBA normalization the parameter $\beta R$ was derived and compared to the full sum-rule fraction of Eq. (1). A correction was then made for the more realis tic Fermi matter distribution according to Bernstein's method. ${ }^{6}$ The values obtained are given in Table III along with data from other probes.

The errors on the EWSR fractions of the LEOR's reflect primarily the uncertainty attached to the continuum subtraction. Consistent with the discussion of the previous section, the errors on the EWSR fraction for ${ }^{75} \mathrm{As},{ }^{96} \mathrm{Mo}$, and ${ }^{100} \mathrm{Mo}$ are larger $( \pm 40 \%)$ due to an increased width and/or decreased strength.

\section{OTHER MULTIPOLES IN THE LEOR REGION}

The excellent quality of the DWBA fits in Fig. 3 ensures the dominance of $l=3$ in the LEOR peak. It is not possible, however, to rule out a moderate contribution from $l=1$ on the basis of the DWBA 
TABLE III. Transition strengths $\left(G_{3}\right)$ and EWSR percentages $(S)$ for the isoscalar octupole strength. Errors on $G_{3}$ and $S$ from the present work (PW) are $\pm 15 \%$ for the lowest $3^{-}$and $\pm 25 \%$ for the LEOR. EWSR percentages from published data are also given.

\begin{tabular}{|c|c|c|c|c|}
\hline Nucleus & $\begin{array}{c}E_{x} \\
(\mathrm{MeV})\end{array}$ & $\begin{array}{c}G_{3} \\
\text { (SPU) }\end{array}$ & $\begin{array}{c}S \\
\% \text { EWSR }\end{array}$ & Ref. \\
\hline${ }^{40} \mathrm{Ca}$ & 3.73 & 18.6 & 10 & PW \\
\hline${ }^{40} \mathrm{Ca}$ & $\begin{array}{l}\text { 11. states from } \\
6.026 \text { to } 9.591 \\
\mathrm{MeV}\end{array}$ & & 9.8 & $\begin{array}{l}\left(p, p^{\prime}\right) E_{p}=35 \mathrm{MeV} \\
\text { Ref. } 9 .\end{array}$ \\
\hline${ }^{40} \mathrm{Ca}$ Total & & & 19.8 & \\
\hline${ }^{58} \mathrm{Ni}$ & $\begin{array}{l}4.47 \\
6.07 \\
6.85 \\
7.55\end{array}$ & $\begin{array}{r}15.0 \pm 3.0 \\
1.5 \pm 0.3 \\
4.2 \pm 0.6 \\
2.6 \pm 0.4\end{array}$ & $\begin{array}{l}10 \\
8\end{array}$ & $\begin{array}{l}\text { From compilation of } \\
\text { Ref. } 6 . \\
\text { From compilation of } \\
\text { Ref. } 6 .\end{array}$ \\
\hline${ }^{58} \mathrm{Ni}$ Total & & & 18 & \\
\hline${ }^{66} \mathrm{Zn}$ LEOR & 6.6 & 10 & 16 & PW \\
\hline${ }^{75}$ As LEOR & 6.5 & $\sim 9$ & $\sim 14$ & PW \\
\hline${ }^{89} \mathrm{Y}$ LEOR & 7.1 & $\sim 13$ & $\sim 20$ & PW \\
\hline${ }^{90} \mathrm{Zr}$ & 2.75 & 13.4 & 7.1 & PW \\
\hline LEOR & 7.2 & 14 & 20 & PW \\
\hline${ }^{90} \mathrm{Zr}$ Total & & & 27 & \\
\hline${ }^{92} \mathrm{Mo}$ & 2.28 & $3.0 \pm 0.3$ & 2.9 & $\begin{array}{l}\text { From compilation of } \\
\text { Ref. } 6 .\end{array}$ \\
\hline LEOR & 6.7 & 16 & 22 & $\mathrm{PW}$ \\
\hline${ }^{92}$ Mo Total & & & 25 & \\
\hline${ }^{96}$ Mo LEOR & 6.2 & $\sim 6$ & $\sim 8$ & PW \\
\hline${ }^{100}$ Mo LEOR & 6.1 & $\sim 5$ & $\sim 6$ & PW \\
\hline${ }^{N a t}$ Ag LEOR & 6.4 & & $\sim 20$ & PW \\
\hline${ }^{116} \mathrm{Sn}$ & 2.25 & 17 & 8 & PW \\
\hline LEOR & 6.5 & 17 & 23 & PW \\
\hline${ }^{116} \mathrm{Sn}$ Total & & & 31 & \\
\hline${ }^{118}$ Sn LEOR & 6.9 & $\sim 14$ & $\sim 20$ & PW \\
\hline${ }^{124} \mathrm{Sn}$ & 2.55 & 7.3 & 4.4 & PW \\
\hline LEOR & 6.2 & 13 & 20 & PW \\
\hline${ }^{124} \mathrm{Sn}$ Total & & & 24 & \\
\hline${ }^{142} \mathrm{Nd}$ & 2.03 & 17.4 & 7.3 & PW \\
\hline LEOR & 6.2 & 17 & 22 & PW \\
\hline${ }^{142} \mathrm{Nd}$ Total & & & 29 & \\
\hline${ }^{144} \mathrm{Sm}$ & 1.81 & 27 & 9.2 & PW \\
\hline $\begin{array}{l}\text { LEOR } \\
{ }^{144} \mathrm{Sm} \text { Total }\end{array}$ & 6.5 & 17 & 21 & PW \\
\hline \multirow[t]{2}{*}{${ }^{154} \mathrm{Sm}$} & 1.01 & & 4 & $\begin{array}{l}\text { From compilation of } \\
\text { Ref. } 6 .\end{array}$ \\
\hline & 1.53 & & 3 & $\begin{array}{l}\text { From compilation of } \\
\text { Ref. } 6 .\end{array}$ \\
\hline \multirow{2}{*}{ LEOR } & 3.7 & 9.9 & 8.1 & $\mathrm{PW}$ \\
\hline & 5.7 & 3.2 & 3.8 & PW \\
\hline${ }^{154} \mathrm{Sm}$ Total & & & 19 & \\
\hline${ }^{197} \mathrm{Au}$ LEOR & 4.1 & 22 & 19 & PW \\
\hline${ }^{208} \mathrm{~Pb}$ & 2.61 & 36 & 20 & PW \\
\hline
\end{tabular}

fits in the angular range from $12^{\circ}$ to $35^{\circ}$. The maxima and minima for $l=1$ occur at angles only $0.5^{\circ}$ smaller than those for $l=3$. Between $3^{\circ}$. and $8^{\circ}$ at $E_{\alpha}=96 \mathrm{MeV}$ there is, however, a significant difference between $l=1$ and $l=3$. The $l=1$ angular distribution has a diffraction minimum which is not present for $l=3$. We have studied the ${ }^{116} \mathrm{Sn}$ $\left(\alpha, \alpha^{\prime}\right)^{116} \mathrm{Sn}$ reaction in this angular range at $E_{\alpha}$ $=96 \mathrm{MeV}$ in order to detect any contribution to the LEOR from $l=1$ [for angles between $7^{\circ}$ and $10^{\circ}$ the LEOR is hidden by the impurity reaction ${ }^{1} \mathrm{H}(\alpha, \alpha){ }^{1} \mathrm{H}$ ]. These data (at laboratory angles of $3.5^{\circ}, 4^{\circ}, 5^{\circ}$, and $6^{\circ}$ ) are displayed along with the $115 \mathrm{MeV}$ data in Fig. 4 in terms of the classical 


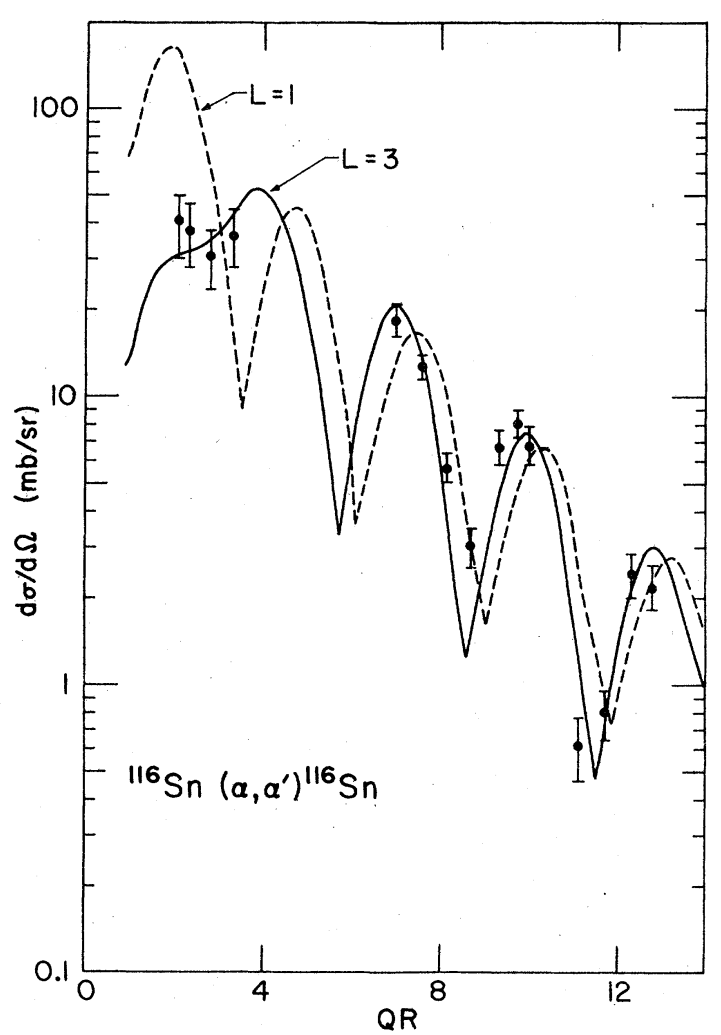

FIG. 4. Angular distribution for the ${ }^{116} \mathrm{Sn}\left(\alpha, \alpha^{\prime}\right)^{116} \mathrm{Sn}$. reaction as a function of $q R$. The curves are DWBA calculations described in the text.

angular momentum transfer $\left|\left(\overrightarrow{\mathrm{k}}_{i}-\overrightarrow{\mathrm{k}}_{f}\right)\right| R$. The DWBA calculations are for $E_{\alpha}=115 \mathrm{MeV}$ and the form factor for $l=1$ was calculated using a model by Satchler. ${ }^{7}$ The bulk of the LEOR is clearly not $l=1$, If it were the ratio $(d \sigma / d \Omega)_{3.5} \circ /(d \sigma / d \Omega)_{6}$ 。 would be $\sim 11$, the experimental value is $1.2 \pm 8$. Detailed studies of the extreme forward angle behavior of various multipoles ${ }^{4}$ has revealed that features such as the $6^{\circ}$ minimum for $l=1$ are very insensitive either to changes in the form factor or to variations in optical-model parameters.

\section{DISCUSSION}

\section{A. LEOR in spherical nuclei}

In the harmonic-oscillator-based schematic model of giant resonances the octupole EWSR strength is divided into two parts, roughly corresponding to $3 \hbar \omega$ and $1 \hbar \omega$ strength. It was shown in Ref. 3 that the LEOR was in good accord with the expected properties of the $1 \hbar \omega$ giant resonance and exhausted roughly $\frac{2}{3}$ ot its expected strength (30-40\% of the total EWSR in the schematic model). Clearly, however, the absence of a strong LEOR in ${ }^{208} \mathrm{~Pb}$ and in ${ }^{40} \mathrm{Ca}$ and lighter mass nuclei cannot be understood in terms of such a simple model. Indeed the presence of at least two

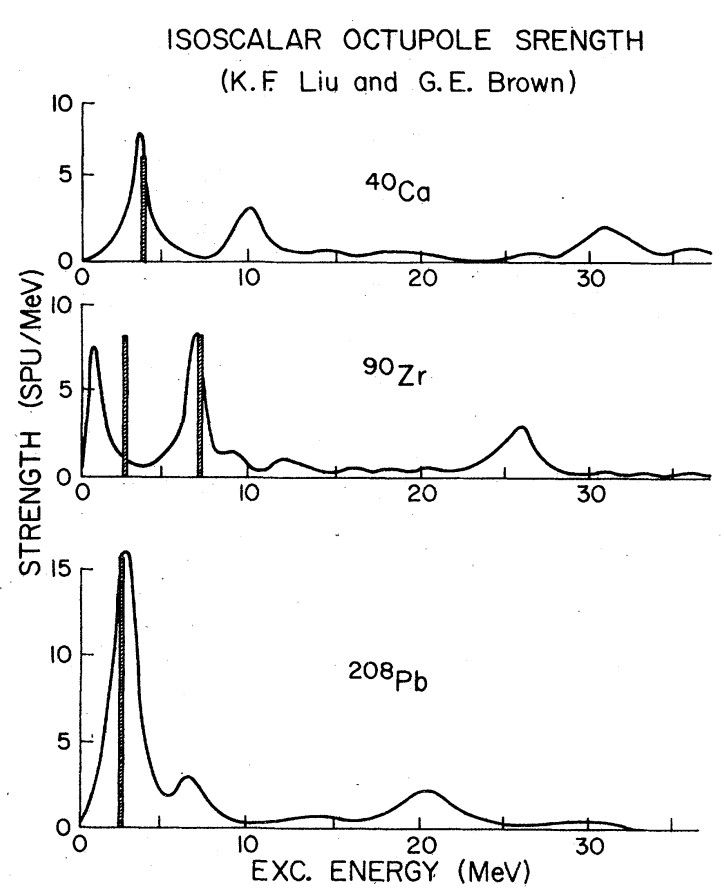

FIG. 5. Theoretical isoscalar octupoie response functions from Ref. 13. The vertical bars are data for lowlying $3^{-}$states and the LEOR.

strong collective $3^{-}$states (the first $3^{-}$and the LEOR) in the region between ${ }^{66} \mathrm{Zn}$ and ${ }^{154} \mathrm{Sm}$ requires a more sophisticated theory. We note that the absence of a LEOR in ${ }^{40} \mathrm{Ca}$ and ${ }^{208} \mathrm{~Pb}$ is not merely the result of relatively poor energy resolution which may miss octupole strength in discrete levels. Very high resolution studies of discrete bound states in ${ }^{40} \mathrm{Ca}$ and ${ }^{208} \mathrm{~Pb}$ have been carried out by investigators at Michigan State University. ${ }^{9,10}$ Although some octupole strength has been seen in these nuclei in the region above the lowest $3^{-}$[there may be a substantial contribution from isovector strength in the $\left(p, p^{\prime}\right)$ reaction], it is very fragmented and therefore unlike the relatively narrow LEOR observed in medium mass nuclei.

Sophisticated continuum RPA calculations have been performed by several author $\mathrm{s}^{12,13,14}$ and compared with considerable success to the properties of other giant resonances. In Fig. 5 we cormpare the calculations of $\mathrm{Liu}$ and Brown ${ }^{13}$ to the present data for the LEOR in ${ }^{90} \mathrm{Zr}$ plus the excitation strengths for the first $3^{-}$states of ${ }^{40} \mathrm{Ca},{ }^{90} \mathrm{Zr}$, and ${ }^{208} \mathrm{~Pb}$. The calculations employed a residual interaction of the Skyrme type. The agreement for ${ }^{90} \mathrm{Zr}$ is excellent; the theory correctly predicts both the location and strength of the LEOR. The excitation energy of the lowest $3^{-}$state is, however, only half of the experimental value. In ${ }^{40} \mathrm{Ca}$ and ${ }^{208} \mathrm{~Pb}$ a rather weak LEOR is indicated theoretically 


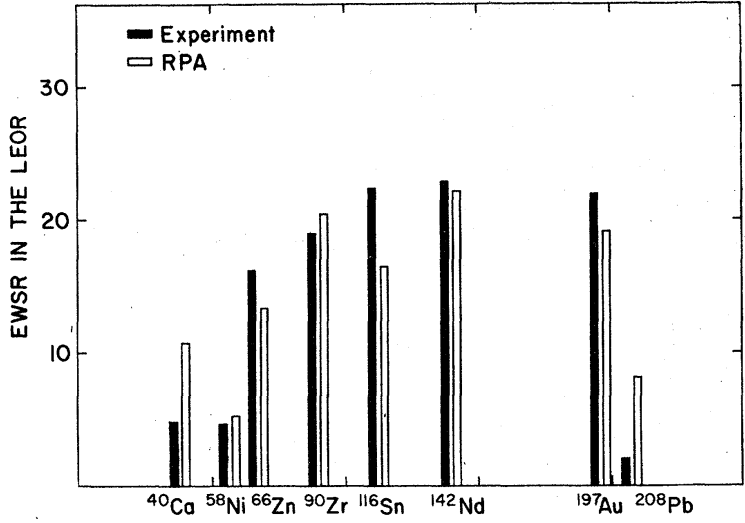

FIG. 6. Comparison of the experimental EWSR strength in the LEOR to that given by RPA calculations described in the text. In ${ }^{40} \mathrm{Ca},{ }^{58} \mathrm{Ni}$, and ${ }^{208} \mathrm{~Pb}$ the solid bars indicate only upper limits on the octupole strength concentrated in a narrow resonance.

which is very suggestive of the experimentally observed absence of a strong resonance. We note that in these two nuclei the low-lying $3^{-}$state already exhausts a large part of the $1 \hbar \omega$ octupole strength. Hence it is not suprising to find a depleted LEOR.

To see whether the RPA can correctly account for the gross properties of the LEOR and low-lying $3^{-}$states in other nuclei, we have performed calculations for ${ }^{40} \mathrm{Ca},{ }^{58} \mathrm{Ni},{ }^{66} \mathrm{Zn},{ }^{116} \mathrm{Sn},{ }^{142} \mathrm{Nd}$, ${ }^{197} \mathrm{Au}$, and ${ }^{208} \mathrm{~Pb}$. The code, written by Kishimoto, ${ }^{15}$ employs a Nilsson-type harmonic-oscillator potential and an octupole-octupole (O-O) residual interaction. The strength of the interaction was chosen by requiring the calculation to correctly reproduce the excitation energy of the first $3^{-}$ state in each nucleus. For ${ }^{197} \mathrm{Au}$ and ${ }^{208} \mathrm{~Pb}$ the strength was determined from the $2.61 \mathrm{MeV}^{-}$in ${ }^{208} \mathrm{~Pb}$. Figure 6 shows the results of the calculations compared to the LEOR data. The marked decrease in strength of the LEOR in ${ }^{208} \mathrm{~Pb}$ and ${ }^{58} \mathrm{Ni}$ is extremely well reproduced. In ${ }^{58} \mathrm{Ni}$ the calculated strength agrees very well with that found in three discrete states. The absence of a LEOR in ${ }^{58} \mathrm{Ni},{ }^{40} \mathrm{Ca}$, and lighter nuclei is probably a result of two factors. First, the RPA predicts a weak LEOR at a modestly high excitation energy [which reduces the $B(E 3)$ for a given EWSR fraction]. Second, the well-known tendency for other giant resonances to become increasingly broad and fragmented in light nuclei may be found for the LEOR as well. The $3^{-}$strength found in the highresolution $\left(p, p^{\prime}\right)$ experiments on ${ }^{40} \mathrm{Ca}$ and ${ }^{24} \mathrm{Mg},{ }^{9,11}$ although reasonably large, does not form a welldefined collective resonance.

The partitioning of EWSR strength between the LEOR and the low-lying $3^{-}$state can vary substan-

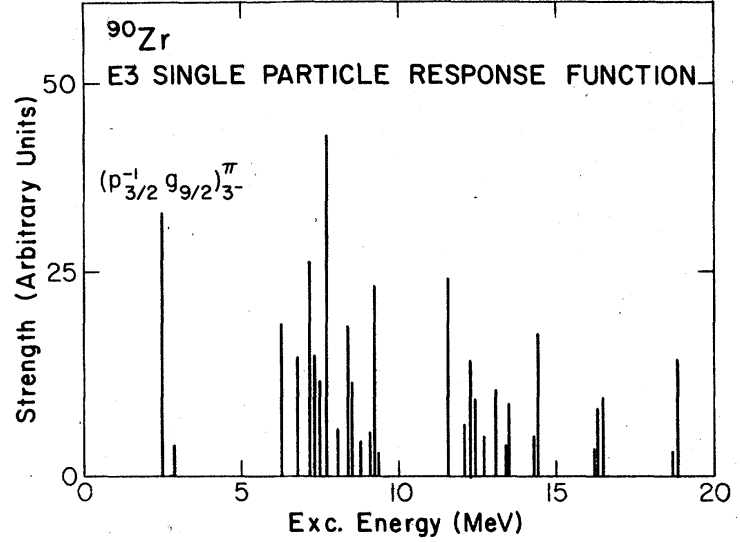

FIG. 7. The unperturbed (no residual interaction) isoscalar octupole response function for ${ }^{90} \mathrm{Zr}$.

tially over a small change in $A$ as it does between ${ }^{66} \mathrm{Zn}$ and ${ }^{58} \mathrm{Ni}$; however, the total $1 \hbar \omega$-strength remains rather constant. The partitioning of the octupole strength, correctly predicted by the RPA, is the result of spin-orbit splitting of the unperturbed octupole response function. This splitting creates certain $1 \hbar \omega$ particle-hole configurations (usually 1 or 2 ) which are considerably below $1 \hbar \omega$. In the case of ${ }^{90} \mathrm{Zr}$, shown in Fig. 7 , it is the $\left(g_{9 / 2}, p_{3 / 2}{ }^{-1}\right)^{\pi}{ }_{3}-$. This low-lying configuration dominates the wave function of the first $3^{-}$state while the LEOR typically contains many components from the other $1 \hbar \omega$ configurations. The balance between these two solutions of the RPA equation depends largely on the initial strength associated with the low-energy configurations. If the low-energy configurations contain much initial strength, as is the case of ${ }^{40} \mathrm{Ca}$ and ${ }^{208} \mathrm{~Pb}$ where both protons and neutrons can contribute, the low-lying $3^{-}$is favored and the LEOR is very weak. In the mass region between $A=66$ and $A=148$ there is typically either a low-energy neutron (near $Z=50$ ) or proton configuration (near $N=50$ or 82 ) but not both; there one sees a strong LEOR containing $\sim \frac{2}{3}$ of the $1 \hbar \omega$ EWSR strength.

A by-product of the fluctuating balance between the LEOR and the first $3^{-}$state may be a residual neutron or proton dominance of the two different octupole regions. Evidence of this is seen in the large difference between the $B(E 3)$ values derived from electron and hadron scattering for the 2.75 $\mathrm{MeV} 3^{-}$in ${ }^{90} \mathrm{Zr}$ (Table IV). The electromagnetic transition (EM) strength is 2.4 times larger than the isoscalar (IS) strength which may indicate the dominance of the $\left(g_{9 / 2}, p_{3 / 2}{ }^{-1}\right)^{\pi}{ }_{3}$-configuration in this state. The IS strength for the lowest $3^{-}$ states in the $\mathrm{Sn}$ isotopes should be larger than the $E M$ values by the same argument; there the dominant configuration is $\left(2 d_{5 / 2}{ }^{-1}, 1 h_{11 / 2}\right)_{3}{ }_{3}$. Unfortu- 
TABLE IV. Comparison of isoscalar (IS) and electromagnetic (EM) transition strengths for lowest $3^{-}$states. SPU denotes single particle units.

\begin{tabular}{cccc}
\hline \hline Nucleus & $\begin{array}{c}E_{\text {exc }} \\
(\mathrm{MeV})\end{array}$ & $\begin{array}{c}G_{3}(\mathrm{IS}) \\
(\mathrm{SPU})\end{array}$ & $\begin{array}{c}G_{3}(\mathrm{EM}) \\
\text { (SPU) }\end{array}$ \\
\hline${ }^{40} \mathrm{Ca}$ & 3.73 & $18.6 \pm 3$ & $31.6 \pm 4 . \mathrm{a}^{\mathrm{a}}$ \\
${ }^{40} \mathrm{Zr}$ & 2.75 & $13.4 \pm 2$ & $32 \pm 5^{\mathrm{a}}$ \\
${ }^{116} \mathrm{Sn}$ & 2.28 & $17 \pm 3$ & $15 \pm 4^{\mathrm{a}}$ \\
& & & $34 \quad \pm 5^{\mathrm{a}}$ \\
${ }^{208} \mathrm{~Pb}$ & 2.61 & $36 \pm 5$ & $39 \pm 5^{\mathrm{a}}$ \\
\hline \hline
\end{tabular}

${ }^{\text {a }}$ Electromagnetic values derived from Ref. 6 .

${ }^{\mathrm{b}}$ Reference 16 .

nately, the large discrepancy in the two $B(E 3)$ 's derived from electron scattering does not permit one to draw a firm conclusion on this point. In addition the large difference between the EM and IS, $B(E 3)$ 's for ${ }^{40} \mathrm{Ca}$ cannot be accounted for by these considerations. Clearly more electromagnetic $B(E 3)$ values must be obtained before the effect of the isospin structure of the experimental probe can be determined reliably.

\section{B. LEOR in deformed and soft-vibrational nuclei}

In the regions of soft-vibrational and deformed nuclei the structure of the GDR undergoes a profound change. Coupling of the dipole mode to lowlying quadrupole degrees of freedom produces a pronounced broadening and/or splitting of the GDR. This is most apparent for vibrational nuclei near $A=75$ and $A=100$ and deformed nuclei between $A=150$ and $A=188$ and those with $A \geqslant 232 .{ }^{17}$ Various collective models have been applied with considerable success to the GDR in these regions. ${ }^{18}$ Contrary to the case of the GDR, the isoscalar GQR shows an extremely small change in width even in permanently deformed nuclei. ${ }^{2,19} \mathrm{~A}$ successful explanation of this qualitatively different behavior has been advanced by Kishimoto et al. ${ }^{19}$. The effect of quadrupole deformation on the LEOR as observed in the Sm isotopes is large and thus more reminiscent of the behavior of the GDR than that of the GQR. In ${ }^{144} \mathrm{Sm}$ and ${ }^{148} \mathrm{Sm}$ the LEOR strength is contained in one peak (with fine structure in the case of ${ }^{144} \mathrm{Sm}$ ) at $E_{x} \sim 6.2 \mathrm{MeV}$. In deformed ${ }^{154} \mathrm{Sm}$ two octupole peaks are seen at $E_{x}=5.7$ and $3.7 \mathrm{MeV}$. The lower of these states has a width of $\sim 2 \mathrm{MeV}$ compared to $\sim 1 \mathrm{MeV}$ for the upper. This suggests that the lower peak may contain at least two of the four expected components of an octupole vibration of an axially symmetric quadrupole deformed nucleus (with angular momentum projections $K=0^{-}$through $3^{-}$). We have also studied the deformed nuclei ${ }^{159} \mathrm{~Tb}$ and ${ }^{174} \mathrm{Yb}$. There, although octupole strength is evi- denced by the phase of certain structures in the continuum spectrum, the distribution is complex and the non-octupole continuum is large. Thus no unambiguous procedure could be found to effect the separation of the octupole strength.

Two theoretical models have been advanced to explain the structure of the LEOR in deformed nuclei。 One, due to Malov et al., ${ }^{20}$ is based on a continuum RPA treatment. The other, by Kishimoto, is an extension of the work of Ref 19 to the octupole case. A central feature of the latter model is a renormalization of the $0-0$ residual interaction which arises from the requirement that the interaction be self-consistent. These models are compared to the data for ${ }^{154} \mathrm{Sm}$ in Fig. 8. Both are in qualitative agreement with the data. Comparison of theory and experiment for a wider range of deformed nuclei is essential if a choice is to be made between the two competing models.

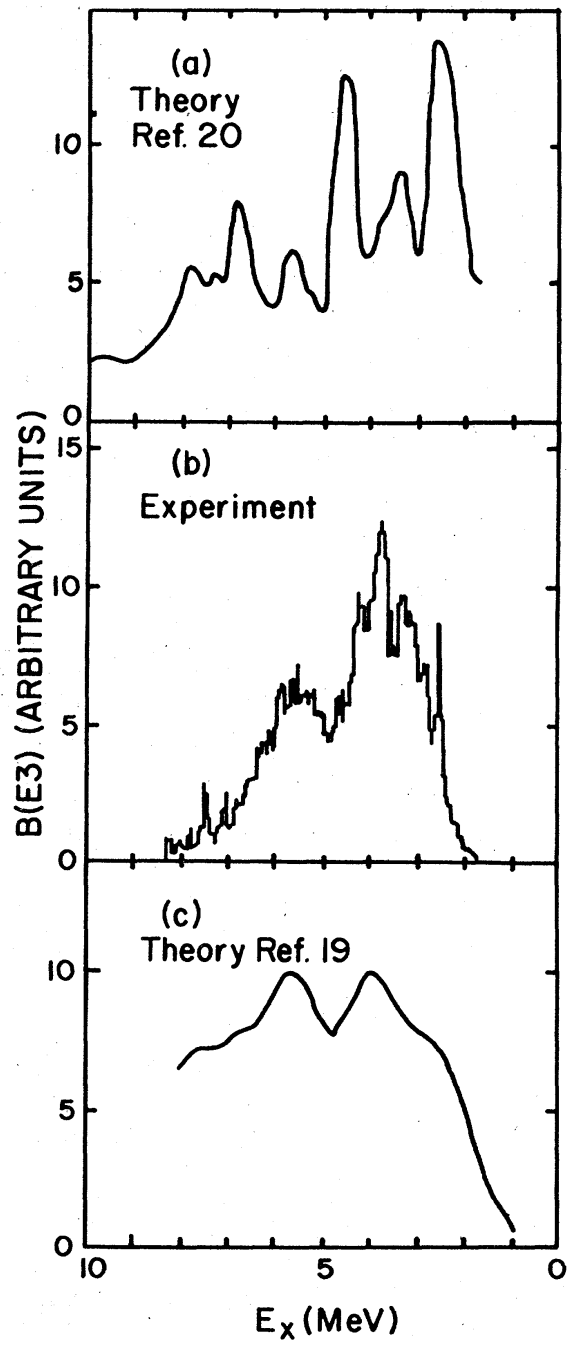

FIG. 8. Comparison of the theories of Refs. 19 and 20 for the LEOR in deformed ${ }^{154} \mathrm{Sm}$. 
The nuclei ${ }^{75} \mathrm{As},{ }^{96} \mathrm{Mo}$, and ${ }^{100} \mathrm{Mo}$ also exhibit a weakening of the LEOR which is likely due to an octupole-quadrupole coupling effect. The peak cross section for the Mo isotopes 92,96 , and 100 are, respectively, 20, 7, and $6 \mathrm{mb} / \mathrm{sr}$. The magnitude of the continuum in ${ }^{96} \mathrm{Mo}$ and ${ }^{100} \mathrm{Mo}$ prevents one from drawing any firm conclusion about the quantitative nature of this process: The resonance may be weaker or merely broader than in ${ }^{92} \mathrm{Mo}$. Our RPA calculations for these three isotopes using a residual interaction derived from the first $3^{-}$in ${ }^{92}$ Mo indicate that the observed weakening is not primarily a simple shell model effect.

\section{CONCLUSION}

The LEOR, a prominent broad peak at $30 / A^{1 / 3}$ $\mathrm{MeV}$ observed in the $(\alpha, \alpha)$ reaction, has been extensively studied and found to be a consistent feature of all nuclei between $A=66$ and $A=197$. It is not seen either in ${ }^{208} \mathrm{~Pb}$ or in ${ }^{40} \mathrm{Ca}$ and lighter nuclei. The fraction of the isoscalar EWSR exhausted varies from $7 \%$ to $23 \%$. If one adds to this the strength contained in the first $3^{-}$state, essentially all of the $1 \hbar \omega$ isoscalar octupole strength can be accounted for in nuclei from ${ }^{40} \mathrm{Ca}$ to ${ }^{208} \mathrm{~Pb}$. The remaining octupole strength $(65-70 \%)$ is expected to exist in a $3^{-}$giant resonance near $E_{x} \sim 100 / A^{1 / 3}$ MeV. Preliminary evidence for such a resonance has been obtained in electron scattering studies. ${ }^{21}$

Comparison of the present data with the continuum RPA calculations of Lix and Brown reveals good agreement for the LEOR in ${ }^{90} \mathrm{Zr}$ and qualita- tive agreement with the experimentally observed absence of the LEOR in ${ }^{40} \mathrm{Ca}$ and ${ }^{208} \mathrm{~Pb}$. Conventional RPA calculations with an adjustable 0-0 residual interaction give an excellent account of the LEOR in the medium mass region and its weakening at the expense of the lowest $3^{-}$state in ${ }^{208} \mathrm{~Pb}$ and in light nuclei. The latter calculations also suggest that substantial differences in the distribution of octupole strength might be observed by probes of different isospin structure due to a dominance of either proton or neutron $\mathrm{p}-\mathrm{h}$ configurations. A promising test of this prediction would be a comparison of accurate electromagnetic and isoscalar $B(E 3)$ 's in nuclei with either closed proton or closed neutron shells.

The coupling between the LEOR and the quadrupole vibrational modes is a likely explanation for the weak LEOR' s seen in ${ }^{75} \mathrm{As},{ }^{96} \mathrm{Mo}$, and ${ }^{100} \mathrm{Mo}$. At present the data on permanently deformed nuclei is too ambiguous to distinguish between competing models of the LEOR in deformed nuclei. Singles $\left(\alpha, \alpha^{\prime}\right)$ experiments in the range of $E_{\alpha}=100$ $\mathrm{MeV}$ are probably inadequate to unambiguously yield the true structure of the octupole response function in soft-vibrational and deformed nuclei. Detection of decay $\gamma$ rays in coincidence with inelastically scattered $\alpha$ particles has not been attempted. Since a large part of the $1 \hbar \omega$ octupole strength lies in the region where particle decay is forbidden or at least inhibited, such experiments are a promising tool for the quantitative elucidation of the properties of the LEOR.
*Supported in part by the National Science Foundation and the Robert A. Welch Foundation.

${ }^{1}$ F. E. Bertrand, Annu. Rev. Nucl. Sci. 26, 457 (1976).

${ }^{2}$ D. H. Youngblood, J. M. Moss, C. M. Rozsa, J. D. Bronson, and A. D. Bacher, Phys. Rev. C $\underline{13}, 944$ (1976).

${ }^{3}$ J. M. Moss, D. H. Youngblood, C. M. Rozsa, D. R. Brown, and J. D. Bronson, Phys. Rev. Lett. $\underline{37}, 816$ (1976).

${ }^{4}$ D. H. Youngblood, C. M. Rozsa, J. M. Moss, D. R. Brown, and J. D. Bronson, Phys. Rev. Lett. 39, 1188 (1977).

${ }^{5}$ K. A. Snover, K. Ebisawa, D. R. Brown, and P. Paul, Phys. Rev. Lett. 32, 317 (1974).

${ }^{6}$ A. M. Bernstein, Adv. Nucl. Phys. 3 , 325 (1969).

${ }^{7}$ G. R. Satchler, Nucl. Phys. A195, 1 (1974); G. R. Satchler, Comm. Nucl. Part. Phys. $\underline{5}, 145$ (1972).

${ }^{8} \mathrm{P}$. D. Kunz, private communication.

${ }^{9}$ C. R. Gruhn, T. Y. T. Kuo, C. J. Maggiore, H. McManus, F. Petrovich, and B. M. Preedom, Phys. Rev. C 6 , 915 (1972).

${ }^{10}$ W. T. Wagner, G. M. Crawley, G. R. Hammerstein, and H. McManus, Phys. Rev, C 12, 757 (1975).

${ }^{11}$ B. Zwieglinski, G. M. Crawley, H. Nann, and J. A. Nolen, Michigan State University report (unpublished).
${ }^{12}$ G. F. Bertsch and S. F. Tsai, Phys. Rep. $\underline{18 C}, 125$ (1975).

${ }^{13}$ K. F. Liu and G. E. Brown, Nucl. Phys. A265, 385 (1976).

${ }^{14}$ S. Krewald, V. Klemt, J. Speth, and A. Faessler, Nucl. Phys. A281, 166 (1976).

${ }^{15} \mathrm{~T}$. Kishimoto, private communication.

${ }^{16}$ P. Barreau and J. B. Bellicard, Phys. Rev. Lett. 19 , 1444 (1967).

${ }^{17}$ B. L. Berman, Lawrence Livermore Laboratory Report No. 78482 (unpublished).

${ }^{18} \mathrm{M}$. Danos, in Proceedings of the International Conference on Photonucleon Reactions and Applications Asilomar, 1973, edited by B. L. Berman (Lawrence Livermore Laboratory, Univ. of California, 1973), p. 43 (and references therein).

${ }^{19}$ T. Kishimoto, J. M. Moss, D. H. Youngblood, J. D. Bronson, C. M. Rozsa, D. R. Brown, and A. D. Bacher, Phys. Rev. Lett. 35, 552 (1975); and T. Kishimoto, private communication.

${ }^{20}$ L. A. Malov, U. O. Nesterenko, and V. G. Soloviev, J. Phys. G 3 , No. 9, L219 (1977).

${ }^{21}$ R. Pitthan, F. R. Buskirk, E. B. Dally, J. N. Dyer, and K. Maruyama, Phys. Rev. Lett. 33, 849 (1974). 